\title{
Anomalously Rapid Tunneling: Charge Transport across Self-Assembled Monolayers of Oligolethylene glycol)
}

\section{Citation}

Baghbanzadeh, Mostafa, Carleen M. Bowers, Dmitrij Rappoport, Tomasz Żaba, Li Yuan, Kyungtae Kang, Kung-Ching Liao, et al. 2017. "Anomalously Rapid Tunneling: Charge Transport Across Self-Assembled Monolayers of Oligolethylene Glycol)." Journal of the American Chemical Society 139 (22) (May 24): 7624-7631. doi:10.1021/jacs.7b02770.

\section{Published Version}

doi:10.1021/jacs.7b02770

\section{Permanent link}

http://nrs.harvard.edu/urn-3:HUL.InstRepos:34708431

\section{Terms of Use}

This article was downloaded from Harvard University's DASH repository, and is made available under the terms and conditions applicable to Open Access Policy Articles, as set forth at http:// nrs.harvard.edu/urn-3:HUL.InstRepos:dash.current.terms-of-use\#OAP

\section{Share Your Story}

The Harvard community has made this article openly available.

Please share how this access benefits you. Submit a story.

Accessibility 


\section{Anomalously Rapid Tunneling: Charge Transport}

\section{across SAMs of Oligoethylene Glycol}

Mostafa Baghbanzadeh, ${ }^{a, \dagger}$ Carleen M. Bowers, ${ }^{a \dagger}$ Dmitrij Rappoport, ${ }^{a}$ Tomasz aba ${ }^{b}$ Li Yuan, ${ }^{a}$ Kyungtae Kang, ${ }^{a}$ Kung-Ching Liao, ${ }^{a}$ Mathieu Gonidec, ${ }^{a}$ Philipp Rothemund, ${ }^{a}$ Piotr Cyganik, ${ }^{b}$ Alan Aspuru-Guzik, ${ }^{a}$ and George M. Whitesides ${ }^{a, c, d} *$

${ }^{a}$ Department of Chemistry and Chemical Biology, Harvard University, 12 Oxford Street, MA 02138

${ }^{b}$ Smoluchowski Institute of Physics, Jagiellonian University, Lojasiewicza 11, 30-348 Krakow, Poland ${ }^{c}$ Kavli Institute for Bionano Science \& Technology, School of Engineering and Applied Sciences, Harvard University, 29 Oxford Street, MA 02138

${ }^{d}$ Wyss Institute of Biologically Inspired Engineering, Harvard University 60 Oxford St. Cambridge, MA 02138, USA

* Author to whom correspondence should be addressed.

${ }^{\dagger}$ Both authors contributed equally to this work.

KEYWORDS: Charge transport; EGaIn; molecular electronics; self-assembled monolayer; ethylene glycol; DFT; superexchange 


\section{ABSTRACT}

This paper describes charge transport by tunneling across self-assembled monolayers (SAMs) of thiolterminated derivatives of oligo(ethylene glycol) $\left(\mathrm{HS}\left(\mathrm{CH}_{2} \mathrm{CH}_{2} \mathrm{O}\right)_{n} \mathrm{CH}_{3} ; \mathrm{HS}(\mathrm{EG})_{n} \mathrm{CH}_{3}\right)$; these SAMs are positioned between gold bottom electrodes and $\mathrm{Ga}_{2} \mathrm{O}_{3} / \mathrm{EGaIn}$ top electrodes and are of the form: $\mathrm{Au}^{\mathrm{TS}} / \mathrm{S}(\mathrm{EG})_{\mathrm{n}} \mathrm{CH}_{3} / / \mathrm{Ga}_{2} \mathrm{O}_{3} / \mathrm{EGaIn}$. Comparison of the attenuation factor $(\beta$ of the simplified Simmons equation) across these SAMs with the corresponding value obtained with length-matched SAMs of $n$ alkanethiols demonstrates, surprisingly, that SAMs of oligoethylene glycol have values of $\beta(\beta=0.29 \pm$ $0.02 \mathrm{n}_{\text {atom }}{ }^{-1}$ and $\left.\beta=0.24 \pm 0.01 \AA^{-1}\right)$ lower than those of SAMs of $n$-alkanethiolates $(\beta=0.94 \pm 0.02$ $\mathrm{n}_{\text {atom }}{ }^{-1}$ and $\beta=0.77 \pm 0.03 \AA^{-1}$ ). The value of $\beta$ for tunneling across oligoethylene glycols is comparable to that across oligophenylenes $\left(\beta=0.28 \pm 0.03 \AA^{-1}\right)$. There are two possible origins for the unexpectedly low value of $\beta$ for $(\mathrm{EG})_{\mathrm{n}}$-derived SAMs. The more probable involves a mechanism for tunneling based on the superexchange model. This model accounts for the rapid hole tunneling across SAMs of oligo(ethylene glycol)s using interactions among the high-energy, occupied orbitals associated with the lone-pair electrons on oxygen. According to calculations using density functional theory (DFT), these orbitals—localized orbitals predominately on the backbone oxygen atoms-are lower in energy $\left(\mathrm{E}_{\mathrm{MO}}=-6.8--7.2 \mathrm{eV}\right)$, but more delocalized (due to interactions between orbitals on neighboring oxygen atoms), than the highest occupied molecular orbital (HOMO, $\mathrm{E}_{\mathrm{MO}}: \sim-5.7 \mathrm{eV}$ ) localized on sulfur. Nonetheless, the existence of these high-energy, delocalized occupied orbitals, which are not present in analogous $n$-alkanethiols $\left(\mathrm{E}_{\mathrm{MO}}<-8.5 \mathrm{eV}\right.$ for orbitals associated with $\left.\mathrm{CH}_{2}\right)$, rationalize the low value of $\beta$. SAMs of oligo(ethylene glycol)s (and of oligomers of glycine). SAMs based on $\mathrm{S}(\mathrm{EG})_{\mathrm{n}} \mathrm{CH}_{3}$ are, in this mechanism, good conductors (by hole tunneling), but good insulators (by electron and/or hole drift conduction)—an unexpected observation that suggests SAMs derived from these or electronically similar molecules as a new class of electronic materials. A second but less probable mechanism for this unexpectedly low value of $\beta$ for $\mathrm{SAMs}$ of $\mathrm{S}(\mathrm{EG})_{\mathrm{n}} \mathrm{CH}_{3}$ rests on the 
possibility of disorder in the SAM, and a systematic discrepancy between different estimates of the thickness of these SAMs. 


\section{INTRODUCTION}

Determining the relationship between the structure of the molecules that make up selfassembled monolayers (SAMs), and the shape of the tunneling barrier that these molecules generate, is an area of active research, and a part of the broader area of "molecular electronics". ${ }^{1-14}$ Our studies have used a junction with the structure M/A-R-T// $\mathrm{Ga}_{2} \mathrm{O}_{3} / \mathrm{EGaIn}$ (where "A" is the group anchoring the molecules of the SAM to the metal "M", "R" is the backbone of the molecule making up the SAM, "T" is the terminal group, and EGaIn is eutectic alloy of gallium and indium. For $n$-alkanethiolates, for example, $\mathrm{A}=\mathrm{S}, \mathrm{R}=\left(\mathrm{CH}_{2}\right)_{\mathrm{n}}$, and $\mathrm{T}=\mathrm{CH}_{3}$ or $\mathrm{H}$, depending on the data being considered. Studies of a range of SAMs based on $n$-alkanethiolates with different terminal groups (both aliphatic and aromatic), and having the same overall length, have shown remarkably little variation in tunneling current; ${ }^{15-16}$ inclusion of groups capable of extended delocalization do lower the barrier to tunneling. ${ }^{17-20}$

This study focuses on the influence of the energy levels of backbone substituents with highlying occupied orbitals on the height of the tunneling barrier and rates of charge transport. We replaced backbone methylene groups $\left(-\mathrm{CH}_{2}-\right)$ with oxygen atoms, and analyzed the influence of this substitution on the rate of charge transport. The work is complementary to our recently described measurements of charge transport by tunneling across SAMs of short oligoglycines, where the presence of consecutive amide $\left(-\left(\mathrm{NHCH}_{2} \mathrm{CO}\right)_{\mathrm{n}}-\right)$ bonds $(\mathrm{n}=1-5)$ in a molecule $\left(\right.$ e.g., $\left.(\mathrm{Gly})_{\mathrm{n}}\right)$ lowered the height of the tunneling barrier relative to length-matched $n$-alkanethiolates, and resulted in a decrease in the measured values of $\beta$ of the simplified Simmons equation from $\beta_{(\mathrm{CH} 2) n}=0.94 \mathrm{n}_{\text {atom }}{ }^{-1}$ to $\beta_{(G l y) n}=0.50 \mathrm{n}_{\text {atom }^{-1}}{ }^{21}$

We measured rates of charge transport across SAMs containing oligomers of ethylene glycol $\left(\mathrm{HS}\left(\mathrm{CH}_{2} \mathrm{CH}_{2} \mathrm{O}\right)_{n} \mathrm{CH}_{3} ; \mathrm{HS}(\mathrm{EG})_{n} \mathrm{CH}_{3}\right)$, and compared these rates to rates across $n$-alkanethiolates matched in the number of atoms along the molecular backbone. Modeling of the oligo(ethylene glycol)s bound to clusters of gold with Density Functional Theory (DFT) predicts a characteristic set of 
high-lying, delocalized, occupied molecular orbitals, which result from a weak coupling between the lone-pair orbitals on neighboring oxygen atoms in the backbone, and which are absent in $n$ alkanethiolates. We associate this change in the electronic topography of the tunneling barrier with a decrease in $\beta\left(\beta_{(C H 2) n}=0.94 \mathrm{n}_{\text {atom }}{ }^{-1}\left(0.77 \AA^{-1}\right)^{22}\right.$ and $\left.\beta_{(E G) n}=0.29 \mathrm{n}_{\text {atom }}{ }^{-1},\left(0.24 \AA^{-1}\right)\right)$; (here $\mathrm{n}_{\text {atom }}$ is the number of non-hydrogen atoms - $\mathrm{C}, \mathrm{N}, \mathrm{O}-\mathrm{in}$ the backbone of the extended oligomer). Corresponding values of $\beta$ for four different classes of compounds are summarized in Table 1. Studying charge tunneling across SAMs of oligo(ethylene glycol)s enabled us to establish that the presence of multiple oxygen atoms in the backbone substantially decreases $\beta$ (relative to SAMs of length-matched $n$ alkanethiolates). We suggest a rationalization of this decrease based on high-energy, delocalized, occupied MOs formed by superexchange interactions between neighboring oxygen lone-pair orbitals (inferred from DFT results) as being the probable origin of this effect. We discuss, but discount, the possibility that an ambiguity in estimating the thickness of the SAMs of $\mathrm{HS}(\mathrm{EG})_{\mathrm{n}} \mathrm{CH}_{3}$ contributes to this estimate of $\beta$.

Charge Transport by Tunneling. Both the orbital energies of the molecules composing the SAM, and the interfaces between the SAM and the electrodes, influence the rate of charge tunneling. ${ }^{7}$ 17, 23-33 The simplified Simmons equation ${ }^{34}$ (eq.1) provides an approximate analytical parameterization of the rate of charge transport across the barrier, and predicts an exponential decay in current density with an increasing width of the tunneling barrier, $d .^{20,35}$ The value of $d$ is often estimated as the fully extended length (in Angstroms) of the molecule from the anchoring atom bound to the bottom electrode to the distal atom in contact with the $\mathrm{Ga}_{2} \mathrm{O}_{3} / \mathrm{EGaIn}$ top electrode, or as the number of repeating units or non-hydrogen atoms in the backbone of the molecule (i.e., the number of carbon atoms in alkanethiols).

$$
J(V)=J_{0}(V) \cdot 10^{-\beta d / 2.303}
$$


The proper definition of $d$ for molecules with multiple possible conformations or molecules in disordered SAMs requires an understanding of how rates of tunneling depend on the conformation of the molecules involved. An important question is: "Does the rate of charge tunneling (assumed to be through bond) depend on the conformation of the molecules through which tunneling occurs?" This problem is one that has not, so far, been solved (or even seriously addressed).

Although the relationship between the structure (molecular and electronic) and shape of the tunneling barrier is not yet completely defined theoretically, the value of $\beta$ can be used to compare charge transport across different molecular systems. The term $J_{0}(\mathrm{~V})$ is an empirical parameterobtained through extrapolation — that represents a more substantial (and less easily simplified and interpreted) set of approximations than does $\beta .{ }^{22,25,36}$ The interpretation of $J_{0}$ across these SAMs in terms of molecular or molecular-orbital structure—especially for molecules that are heterogeneous in molecular orbital structure (e.g., $(\mathrm{Gly})_{\mathrm{n}}$ and $\left.(\mathrm{EG})_{\mathrm{n}}\right)$ - is not, at present, straightforward, and—for molecules with structures more complex than $n$-alkanethiols—extrapolation to $d=0$ will not yield a meaningful value; we therefore focus our analysis on differences in $\beta$.

\section{Superexchange Model of Hole Tunneling through Oligo(Ethylene Glycol)s. The}

superexchange model ${ }^{37-39}$ is a simple atomistic model of tunneling through molecules. This tunneling model predicts an exponential dependence of current density with the length of the molecule, and is compatible with both the simplified Simmons equation (Eq. 1) and empirical calculations. ${ }^{34}$ It considers the molecule as a one-dimensional chain of localized orbitals with relatively weak superexchange couplings between them, usually due to interactions between neighboring groups of the same type. ${ }^{37-38,40}$ Specifically, in this work, these localized orbitals are oxygen lone pairs in oligo(ethylene glycol)s and peptide bond orbitals in oligo(glycine)s. The superexchange model was developed by McConnell, Ratner, Nitzan, and coworkers ${ }^{37-39}$ to describe charge transport in peptides, ${ }^{41}$ and generally provides a qualitative model for electronic conduction (hopping and tunneling) across 
molecular systems. Because it is conceptually simple, it also provides an interpretable relationship between the electronic structures of the molecules and their tunneling properties. Perhaps even more importantly, it provides a framework with which to compare different classes of molecules.

We consider a linear chain of $n$ identical units, with one localized orbital per unit, interacting via superexchange with the localized orbitals of the units to its immediate left and right. The (hypothetical) energy of each localized orbital in the absence of the couplings is called the local orbital energy $\varepsilon_{0}$, while the coupling strength between orbitals (in units of energy) is denoted as $V .{ }^{42}$ Then, the orbital energies $\varepsilon_{j}$ of the MOs resulting from superexchange couplings follow a characteristic splitting pattern, ${ }^{37-39}$

$$
\varepsilon_{j}=\varepsilon_{0}+2 V \cos \left(\frac{j \pi}{n+1}\right), j=1, \ldots, n,
$$

where the index $j$ counts the MOs in the order of increasing energy, $n$ is the total number of units in the chain, and $\pi$ is the circle constant The local orbital energy (in the absence of coupling) $\varepsilon_{0}$, and the coupling strength $V$ are defined above. By equating the predicted orbital energies $\varepsilon_{j}$ with the energies of a set of molecular orbitals (MOs) obtained from DFT calculations, we are able to extract the determining parameters $\varepsilon_{0}$ and $V$.

We have previously applied this model quantitatively to rationalize the rapid rate of tunneling across SAMs of oligoglycines relative to $n$-alkanethiolates. ${ }^{21}$ This model suggested that the substantial couplings between the high-energy occupied orbitals (through which hole tunneling occurs) on the neighboring amide bonds of the oligoglycine molecules were responsible for the experimentally observed rapid (relative to $n$-alkanethiolates) rate of charge transport. ${ }^{21}$ As predicted by Ratner, Nitzan, and coworkers, high tunneling rates are achieved by i) reducing the energy difference between the local orbital energy $\varepsilon_{0}$ and the Fermi level of the metal $\varepsilon_{\mathrm{F}}$, and ii) increasing the coupling strength $V .{ }^{37-38,40}$ Here, we investigate the applicability of the superexchange model to hole tunneling across SAMs of oligo(ethylene glycol)s, and estimate the corresponding values of the parameters $\varepsilon_{0}$ and $V$ from the 
orbital energies of the MOs computed by DFT. (The Supporting Information gives details of the DFT calculations and the parameters of the superexchange model.)

Structure of SAMs of Oligo(Ethylene Glycol)s. We formed thiolated monolayers composed of molecules of oligo(ethylene glycol)s $\left(\right.$ e.g., $\left.\mathrm{HS}(\mathrm{EG})_{\mathrm{n}} \mathrm{CH}_{3}\right)$, and characterized SAMs of them $(n=1$ 7), on surfaces of template-stripped ${ }^{43}$ gold $\left(\mathrm{Au}^{\mathrm{TS}}\right)$ using XPS, IRRAS, and static contact angles with water. We relied on XPS to calculate the thickness of the SAMs of $\mathrm{HS}(\mathrm{EG})_{\mathrm{n}} \mathrm{CH}_{3}$ on $\mathrm{Au}^{\mathrm{TS}}$, and compared these values of thickness to lengths calculated assuming an all-trans extended conformation and an all-helical conformation (Figure S4). When $n=2$ and 3, the values of thickness are comparable to the all-trans extended lengths. When $n>3$, the thicknesses are comparable to the lengths calculated for a helical conformation, suggesting a possible transition in structure with increasing lengths units of ethylene glycol. This suggestion is in agreement with observations from Vanderah et al. ${ }^{44-45}$ The results from our structural analysis using IRRAS indicates the formation of non-crystalline structures of chemisorbed molecules of ethylene glycol on the surface over the entire range of $n$. The heterogeneous structural conformation observed in the SAMs of ethylene glycol produce variability in surface coverage and effective film thickness, both of which are lower than the corresponding values for highly-ordered SAMs, such as $n$-alkanethiolates of similar molecular length. The details of our structural analysis are in the Supporting Information.

Meuse and coworkers studied the structural variation and ordering of SAMs of oligo(ethylene glycol)s $\left(\mathrm{HS}(\mathrm{EG})_{\mathrm{n}} \mathrm{CH}_{3}\right.$, where $n=3-6$, on $\left.\mathrm{Au}\right)$ for potential applications in the generation of protein resistant surfaces. Using reflection-absorption infrared spectroscopy, spectroscopic ellipsometry, and electrochemical impedance spectroscopy, ${ }^{44-45}$ their results suggest that monolayers of oligo(ethylene glycol)s on Au are oriented normal to the surface, regardless of the number of units of ethylene glycol (from $n=3-6$ ). When $n<5$, they observed the formation of amorphous structures with some all-trans 
conformations, but little evidence of helical conformations. When $n=5$ or 6 , they observed the formation of highly-ordered helical structures. ${ }^{45}$

The upshot of these studies is that there is not uniform agreement in the literature about the conformation of the $(\mathrm{EG})_{\mathrm{n}}$ groups in SAMs. Our XPS work, carried out with modern instruments (Thermo Scientific K-Alpha XPS) with a good signal to noise ratio, clearly indicates conformations that extend approximately perpendicular to the surface. Analysis by ellipsometry indicates thinner monolayers than expected based on calculated lengths, but accurate ellipsometry on these very thin

films is difficult, ${ }^{7,46}$ and we conclude that the values calculated by XPS are correct for our system.

The influence of trans extended vs. helical conformation on the orbital energy is not clear. DFT calculations show that the local orbital energies $\varepsilon_{0}$ of oxygen lone pairs and the coupling strengths $\mathrm{V}$ are not significantly different between the trans-extended and all-helical conformations of oligo(ethylene glycol) chains (See Supporting Information).

\section{RESULTS AND DISCUSSION}

Formation of SAMs. We formed SAMs of thiol-terminated oligo(ethylene glycol)s on templatestripped gold substrate $\left(\mathrm{Au}^{\mathrm{TS}}\right)^{47}$ by immersing the $\mathrm{Au}^{\mathrm{TS}}$ substrates in a 3-mM ethanolic solution ${ }^{44}$ of thiol, purged with nitrogen, for 24 hours at room temperature. Immediately prior to electrical measurements, we rinsed the SAMs with ethanol, and removed the residual ethanol by evaporation under a gentle stream of nitrogen. Table S6 summarizes the results for charge tunneling across SAMs of oligo(ethylene glycol)s and $n$-alkanethiolates on $\mathrm{Au}^{\mathrm{TS}}$ using what we call "selected"48 conical tips of $\mathrm{Ga}_{2} \mathrm{O}_{3} / \mathrm{EGaIn}$; we have described this type of junction in detail previously. ${ }^{22}$ Measurements of $J(\mathrm{~V})$ at forward and reverse biases $( \pm 0.5 \mathrm{~V}$ and $\pm 1.0 \mathrm{~V})$ produced nearly symmetrical profiles with rectification ratios $(r=|J(+\mathrm{V}) / J(-\mathrm{V})|) \leq \sim 4$ for both SAMs of alkanethiolates and SAMs of oligo(ethylene glycol)s on gold. Using our junction, this value of $r$ is not large enough to be interpreted, and not statistically distinguishable from 1. 
Measuring Current Densities by Tunneling. Our studies were carried out with junctions of the form $\mathrm{Au}^{\mathrm{TS}} \mathrm{SR} / / \mathrm{Ga}_{2} \mathrm{O}_{3} / \mathrm{EGaIn}$. Experimental studies of charge transport across SAMs of alkanethiolates using this junction and others have been described extensively, ${ }^{22,49-50}$ and the data from these studies on gold serve as standards to which we compare changes in rates of charge transport with changes in molecular structure for other series of compounds (Table 1). (In the study described here, the structural change is the substitution of oxygen for a methylene group). For all systems, Gaussian-mean values of $<\log |J|>$ decreased linearly with increasing $d$ (estimated by assuming a trans-extended conformation for $(\mathrm{EG})_{\mathrm{n}}$ group. Given the complexity in the structure of SAMs of oligo(ethylene glycol)s, we also approximated $d$, the distance between the electrodes, as the number of non-hydrogen atoms in the backbone of the molecules.

Linear regression analyses of the values of $\langle\log |J|>$ versus $d$ yielded values of $\beta$ for SAMs of oligo(ethylene glycol)s on gold substrates (Figures 2 and S9). The value of $\beta$ for SAMs of $\mathrm{HS}(\mathrm{EG})_{\mathrm{n}} \mathrm{CH}_{3}$ was $\beta_{(E G) n}=0.29 \pm 0.02 \mathrm{n}_{\text {atom }}{ }^{-1}$ when assuming $d$ to be the number of non-hydrogen atoms in the backbone of molecule; this value is much lower than that measured for SAMs of $n$ alkanethiolates on gold $\left(\beta_{(\mathrm{CH} 2) n}=0.94 \mathrm{n}_{\text {atom }}{ }^{-1}\right) .{ }^{50}$ We also approximated $d$ as the length of the transextended molecule from the anchoring atom to the distal hydrogen atom to calculate $\beta$ in units of $\AA^{-1}$; this analysis yields $\beta_{(E G) n}=0.24 \AA^{-1}$ (Figure $2 \mathrm{~b}$ ), which is comparable to that describing tunneling across SAMs of oligophenyls on gold $\left(\beta_{(\mathrm{CH} 2) n}=0.28 \AA^{-1}\right) .{ }^{17}$ Figure $\mathrm{S} 9$ shows analyses of $\langle\log |J|>$ versus $d$, where $d$ is approximated as the film thickness (measured with angle-resolved XPS); this method gives $\beta_{(E G) n}=0.54 \AA^{-1}$. Table 1 summarizes $\beta$ and $\log \left|J_{0}\right|$ values for SAMs of oligo(ethylene glycol)s and compares them with other known systems.

The disorder in the molecular structure of SAMs of oligo(ethylene glycol)s, and the probable involvement of multiple conformations, makes it difficult to estimate the width of the tunneling barrier (d) unambiguously. Moreover, it makes the values of rates of charge transport less certain than those 
estimated for SAMs of well-ordered $n$-alkanethiols. Despite this heterogeneity in structure, there is a clear linear dependence of $\langle\log |J|\rangle$ on the value of $d$, when $d$ is approximated either as the number of non-hydrogen atoms along the backbone (Figure 2a) or by estimation for a trans-extended conformation (Figure 2b). This observation suggests that—for charge transport by hole tunneling across SAMs of oligo(ethylene glycol)s — the atomic length of molecules (e.g., the number of atoms in the chain) dominates the width of the tunneling barrier, and not the defects of conformation or regularity of packing of these molecules in the SAM.

To further examine the influence of the structure of SAMs of oligo(ethylene glycol)s on current density, we compared the current density across SAMs of $\mathrm{HS}(\mathrm{EG})_{\mathrm{n}} \mathrm{CH}_{3}$ on $\mathrm{Au}^{\mathrm{TS}}$ with i) $\mathrm{HS}(\mathrm{EG})_{\mathrm{n}} \mathrm{CH}_{3}$ $(\mathrm{n}=3,5)$ on template stripped silver $\left(\mathrm{Ag}^{\mathrm{TS}}\right)$ and ii) $\mathrm{HOOC}(\mathrm{EG})_{\mathrm{n}} \mathrm{CH}_{3}{ }^{51}(\mathrm{n}=2,3)$ on $\mathrm{Ag}^{\mathrm{TS}}($ Figures S7 and S8). Changing the substrate from gold to silver and the anchoring group from thiol to carboxylate influences the structure of the SAM.$^{52}$ Despite the change in substrate and anchoring group, molecules of oligoethylene glycol, with equivalent number of repeating units, produce comparable current densities. The similar current densities between the three classes of SAMs on silver and gold support the conclusion that the length of the oligoethylene glycol molecule-rather than the details of its conformation—defines the width of the tunneling barrier.

The difference in rates of charge transport between SAMs of ethylene glycol and SAMs of alkanethiolate is unexpected given that both molecular systems (structures having both C-C and C-O bonds) have electronic transitions that occur only at wavelengths less than $200 \mathrm{~nm},{ }^{53-54}$ with corresponding HOMO-LUMO gaps for both types of structures of about 7-8 eV, as calculated in the gas phase (Tables S10 and S11). ${ }^{54}$

Computed Molecular Orbital Structures. The differences in the structures of the molecular orbitals from DFT calculations provide a rationalization for the change in $\beta$. DFT calculations using the Becke 3-parameter hybrid exchange-correlation functional (B3LYP) ${ }^{55}$ and split-valence basis sets 
(def2-SVP) $^{56}$ (see Supporting Information for full computational details) indicate that the HOMO is localized on the sulfur anchoring atom in both alkanethiolates and thiolate-terminated oligo(ethylene glycol)s bound to clusters of gold; the energy of this orbital for the two structures is indistinguishable ( -5.6 eV, Table 2). Also, the high-lying MOs located on the $\mathrm{C}-\mathrm{C} \sigma$ bonds of the backbone are similar for both structures $(\sim-8.0 \mathrm{eV}$, Table 2$)$. The notable differences in the computed molecular orbital structures are due to oxygen lone-pair orbitals, which give rise to a set of high-energy occupied MOs delocalized over the oligoethylene glycol backbone. The highest-energy MO for each oligoethylene glycol chain length is shown in Table 2.

Our crucial observation is that the orbital energies of these MOs formed from oxygen lone-pair orbitals computed using DFT follow the functional relationship predicted by the superexchange tunneling model (eq.2) with good accuracy. By fitting the orbital energies $\varepsilon_{j}$ of these MOs in oligo(ethylene glycol)s $\left(\mathrm{S}(\mathrm{EG})_{n} \mathrm{CH}_{3}, n=3-7\right)$ bound to clusters of gold $\left(\mathrm{Au}_{10} \mathrm{~S}(\mathrm{EG})_{n} \mathrm{CH}_{3}\right)$ against their orbital indices $j$ and the chain lengths $n$, we are able to extract the determining parameters of the superexchange model: the (hypothetical) energy of an isolated oxygen lone-pair orbital (local orbital energy) $\varepsilon_{0}=(-6.99 \pm 0.007) \mathrm{eV}$ and the coupling strength $V=(-0.09 \pm 0.005) \mathrm{eV}$ between the oxygen lone pairs at neighboring ethylene glycol units. The Supporting Information describes the DFT calculations and the fit to the superexchange model in more detail.

Superexchange Model of Tunneling in Oligo(Ethylene Glycol)s. The oxygen lone pairs have a (hypothetical) orbital energy of $\sim-7.0 \mathrm{eV}$ in the absence of superexchange interactions; this value is still well below the HOMO energy of $\sim-5.6 \mathrm{eV}$. The effect of the superexchange coupling is, however, to create a set of high-energy, delocalized, occupied MOs which extend along the molecular backbone (unlike the HOMO which is localized predominately on the sulfur atom; Table 2). We note that the interaction between occupied orbitals (oxygen lone pairs) does not fit the textbook picture of bond formation due to overlap between an occupied and a vacant orbital. The coupling strength of the 
interaction between oxygen lone pairs $(|V| \sim 0.1 \mathrm{eV})$ is less than $5 \%$ of the bond energies of typical bonds $(2-4 \mathrm{eV})$ and does not contribute to the stabilization (or destabilization) of the oligo(ethylene glycol) chains. Rather, it is due to a relatively weak quantum mechanical (exchange) effect between the electrons occupying the oxygen lone-pair orbitals on the neighboring ethylene glycol units.

This proposal for an electronic basis for rapid hole tunneling is similar to that which we proposed for hole tunneling across SAMs of oligoglycine, ${ }^{21}$ and the two studies are thus mutually reinforcing, especially since they involved two classes of molecules with very different, molecular and electronic structures. Studying the electronic structures of oligoether chains of different composition gives us an opportunity to investigate how the distance between the oxygen atoms along the molecular backbone influences the molecular electronic parameters relevant to hole tunneling. To this end, we compared the results of the DFT calculations for oligo(ethylene glycol)s bound to a (Au) ${ }_{10}$ cluster with those for oligo(1,3-propanediol) $\left(\mathrm{Au}_{10} \mathrm{~S}\left(\mathrm{CH}_{2} \mathrm{CH}_{2} \mathrm{CH}_{2} \mathrm{O}\right)_{n} \mathrm{CH}_{3} ; n=3-5\right)$ and oligo(butane-1, 4-diol) $\left(\mathrm{Au}_{10} \mathrm{~S}\left(\mathrm{CH}_{2} \mathrm{CH}_{2} \mathrm{CH}_{2} \mathrm{CH}_{2} \mathrm{O}\right)_{n} \mathrm{CH}_{3} ; n=3-5\right)$ similarly bound to gold $\left(\mathrm{Au}_{10} \mathrm{~S}\left(\mathrm{CH}_{2} \mathrm{CH}_{2} \mathrm{CH}_{2} \mathrm{O}\right)_{n} \mathrm{CH}_{3} ; n=1-\right.$ 3). Surprisingly, the coupling strength in the series of gold-bound oligo(1,3-propanediol)s is indistinguishable from that in oligo(ethylene glycol)s, despite the larger distance between the oxygen atoms: $\varepsilon_{0}=(-6.91 \pm 0.004) \mathrm{eV}, V=(-0.09 \pm 0.003) \mathrm{eV}$. Four $-\mathrm{CH}_{2}-$ units between the oxygen atoms are necessary to reduce the coupling strength to a value that is effectively "non-interacting;" the series of gold-bound oligo(1,4-butanediol) give $\varepsilon_{0}=(-6.85 \pm 0.01) \mathrm{eV}$ and $V=(-0.05 \pm 0.01) \mathrm{eV}$ (Figures S12 and S13).

\section{CONCLUSION}

The interaction of multiple oxygen atoms along the backbone of SAMs of oligo(ethylene glycol)s ( $\left.\mathrm{HS}(\mathrm{EG})_{\mathrm{n}} \mathrm{CH}_{3},(\mathrm{n}=1-7)\right)$ influences rates of charge transport by hole tunneling. The value of $\beta$ of the simplified Simmons equation (Table 1) is lower for SAMs of oligo(ethylene glycol)s on gold than for SAMs of $n$-alkanethiolates on gold, and comparable to that of oligophenylenes $\left(-\mathrm{C}_{6} \mathrm{H}_{4}-\right)_{\mathrm{n}}$. The 
oxygen atoms in the backbone interact in a way that result in high-lying, delocalized molecular orbitals at $\sim-7 \mathrm{eV}$ that arise from superexchange coupling between neighboring lone-pair orbitals on oxygen. (The orbitals localized on sulfur and on the $\mathrm{C}-\mathrm{C}$ bonds are unaffected, and their energies on molecules of oligo(ethylene glycol)s and alkanethiolates are indistinguishable.) The strength of the interactions between nearest oxygen atoms in oligoethylene glycols and oligo(1,3-propanediol)s is indistinguishable; this observation suggests that similar measurements in rates of charge transport would be observed.

The strength of the interactions between nearest oxygen atoms calculated using the superexchange model also rationalizes the high tunneling conductivity observed for oligoglycines. ${ }^{21}$ Charge tunneling across SAMs of oligoglycine is comparable to SAMs of oligophenyls ${ }^{21}$, and correlates with the presence of high-lying, delocalized, occupied orbitals formed from the $\pi$ orbitals of the peptide bonds.

The rapid rate of tunneling across SAMs of both oligoethylene glycol and oligoglycine suggests that these classes of molecules should be considered as members of a new class of organic electronic materials: good conductors by tunneling, but poor conductors by electronic drift. The conductivity of these compounds also relevant to questions concerning the ability of biopolymers (both proteins and nucleic acids) to support charge tunneling. Moreover, the observation that calculated interactions between neighboring amide bonds correlate with experimentally observed rates of charge transport suggests that the superexchange model will be useful as a predicative tool in designing molecules with tunneling barriers engineered for potential and topography.

\section{ASSOCIATED CONTENT}

\section{Supporting Information}


Detailed experimental procedure and histograms of current densities. This material is available free of charge via the Internet at http://pubs.acs.org.

\section{AUTHOR INFORMATION}

\section{Corresponding Author}

*gwhitesides@gmwgroup.harvard.edu

\section{Notes}

The authors declare no competing financial interest.

\section{ACKNOWLEDGEMENT}

This work was supported by a subcontract from Northwestern University from the United States Department of Energy (DOE, DE-SC0000989), a grant from the National Science Foundation (NSF, CHE-1506993), and a grant from the National Science Centre Poland (DEC-2013/10/E/ST5/00060). The DOE grant from Northwestern supported the salary for C.M.B, M.B, and K.-C.L in carrying out the experimental design, synthesis, and measurements of tunneling current. The NSF grant supported the salary for Y.L and M.B. for work characterizing the structure of the SAMs. P.R. acknowledges support from MRSEC (DMR 14-20570) in characterizing the EGaIn tips used in the electrical junction. M. G. acknowledges support from Marie Curie IOF FP7 for project SAM-TunEGaIn (PIOF-GA-2012328412) for DFT analysis. D.R and A. A.-G. acknowledge the Cyberdiscovery Initiative Type II (CDI2) grant from the National Science Foundation (NSF, OIA-1125087) for DFT analysis. Sample characterization was performed in part at the Center for Nanoscale Systems (CNS) at Harvard University, a member of the National Nanotechnology Infrastructure Network (NNIN), which is supported by the National Science Foundation (ECS-0335765). We particularly appreciate the assistance of Dr. Hao-Yu Lin at CNS with XPS analysis. 
a)

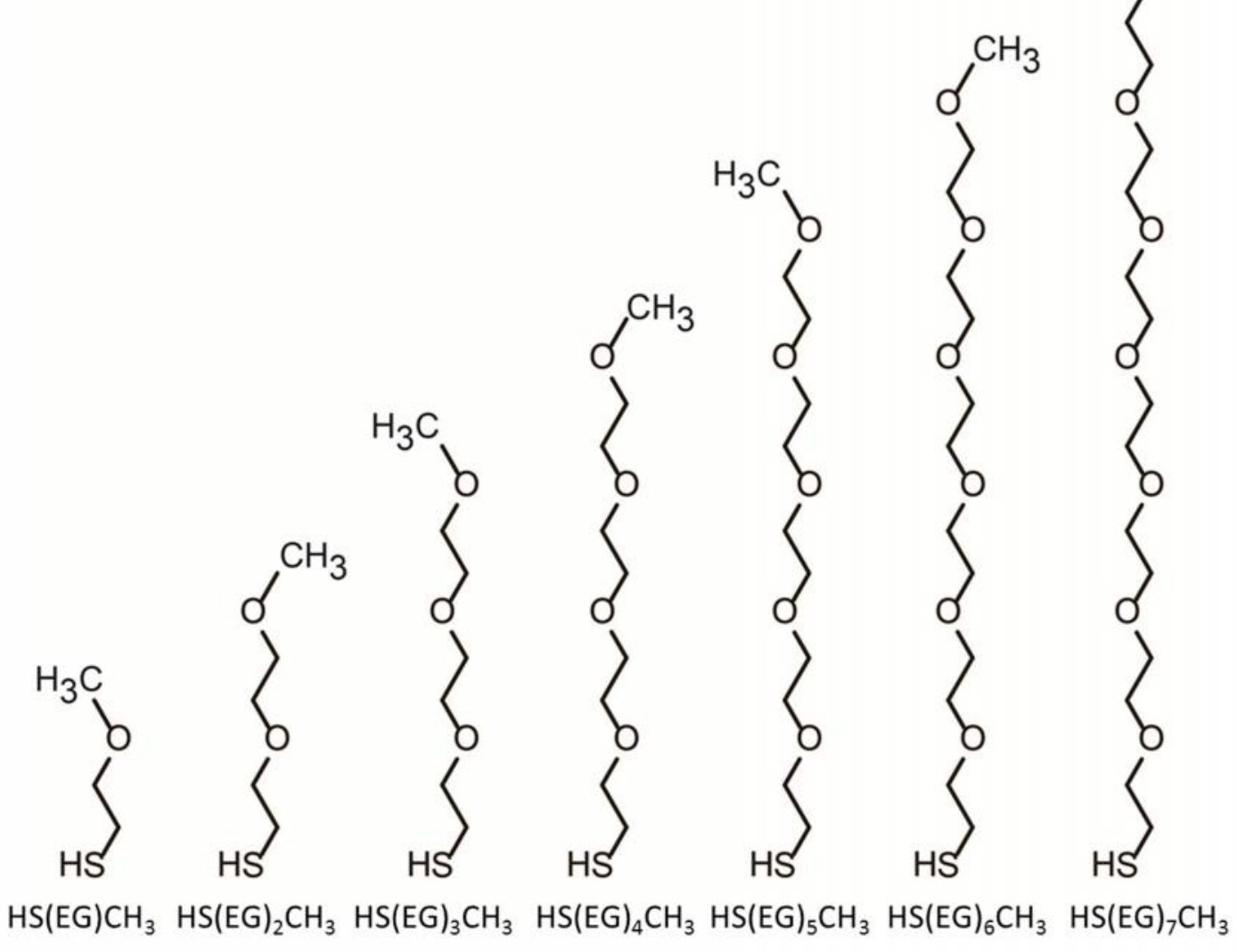

b)

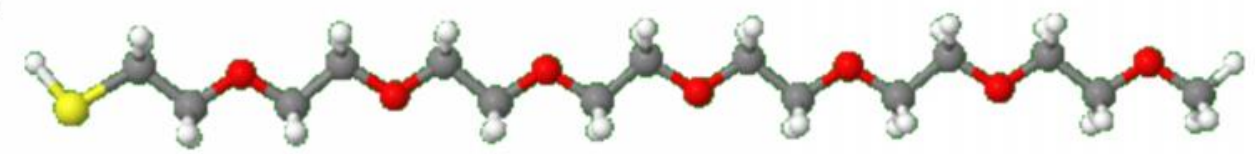

c)

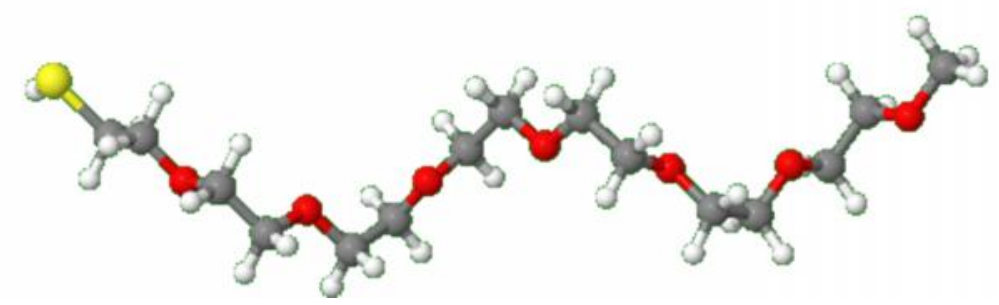

Figure 1. (a) Trans-extended representation of mercapto-oligoethylene glycols used to form SAMs on $\mathrm{Au}^{\mathrm{TS}}$. "EG" indicates ethylene glycol; $\mathrm{n}=$ number of EG units. When the molecules of oligo(ethylene glycol)s are present in a SAM, their structure is non-crystalline, and adopts both (b) all-trans and (c) all helix conformations. The depicted conformation was calculated using DFT with B3LYP and def2-SVP functional, resolution-of-the-identity approximation (RIJ). 

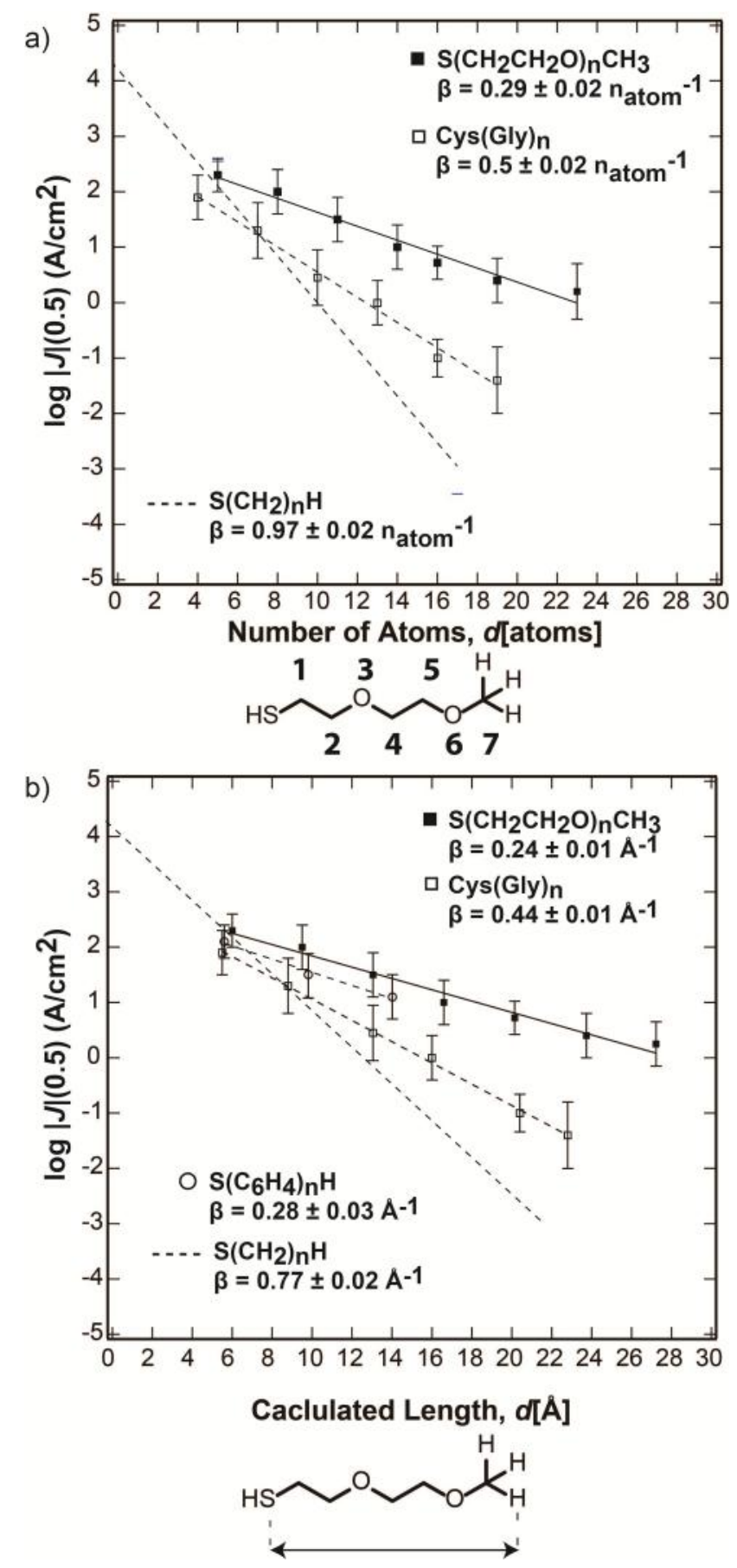

Figure 2. Plot of the Gaussian mean values of $\log |J|$ at $+0.5 \mathrm{~V}$ versus a) the number of non-hydrogen atoms in the backbone of the molecule, starting with carbon next to the sulfur atom, and counting to the final non-hydrogen atom (for example, number of atoms for $\mathrm{HS}(\mathrm{EG})_{2} \mathrm{CH}_{3}$ is 7), and b) the calculated length in $\AA$ for an all trans-extended conformation from the anchoring sulfur atom that chemically contacts to the surface of metal substrates to the distal hydrogen atom. The solid line indicates data collected for oligo(ethylene glycol)s where $n$ is the number of ethylene glycol units in $\mathrm{HS}(\mathrm{EG})_{\mathrm{n}} \mathrm{CH}_{3}$. The dashed line indicates data collected for $n$-alkanethiolates ${ }^{50}$ on $\mathrm{Au}^{\mathrm{TS}}$. The error bars represent the standard deviation of the Gaussian mean values (for details of measurements see Supporting Information). 
Table 1. Summary of values of $\beta$ and $J_{0}$ for different classes of SAMs measured on $\mathrm{Au}^{\mathrm{TS}}$ surfaces. The symbol \pm indicates the error calculated from the standard deviations of the Gaussian mean. ${ }^{17,21,23}$

\begin{tabular}{|c|c|c|c|c|}
\hline \multirow[b]{2}{*}{$\begin{array}{l}\text { Thiolated SAM on } \\
\text { Au }\end{array}$} & \multirow[b]{2}{*}{ Structure } & \multicolumn{2}{|l|}{$\beta$} & \multirow[b]{2}{*}{$J_{0}\left(\mathrm{~A} / \mathrm{cm}^{2}\right)^{\mathrm{c}}$} \\
\hline & & $\AA^{-1 a}$ & $\mathbf{n}_{\text {atom }}{ }^{-1 b}$ & \\
\hline n-Alkane & $\mathrm{HS}\left(\mathrm{CH}_{2}\right)_{\mathrm{n}} \mathrm{H}$ & $0.77 \pm 0.03^{d}$ & $0.94 \pm 0.03$ & $4.2 \pm 0.2$ \\
\hline Oligophenyl & $\mathrm{HS}(\mathrm{Ph})_{\mathrm{n}} \mathrm{H}$ & $0.28 \pm 0.03^{\mathrm{e}}$ & $0.30 \pm 0.03^{\mathrm{f}}$ & $2.7 \pm 0.1$ \\
\hline Oligoglycine & $\mathrm{HS}\left(\mathrm{CH}_{2}\right)_{2}(\mathrm{Gly})_{\mathrm{n}} \mathrm{H}$ & $0.45 \pm 0.02^{\mathrm{g}}$ & $0.50 \pm 0.02$ & $2.8 \pm 0.3$ \\
\hline Oligoethylene glycol & $\mathrm{HS}(\mathrm{EG}){ }_{\mathrm{n}} \mathrm{CH}_{3}$ & $0.24 \pm 0.01$ & $0.29 \pm 0.02$ & $2.9 \pm 0.1$ \\
\hline
\end{tabular}

${ }^{a}$ Length $(d)$ calculated in $\AA$ for an all trans-extended conformation from the anchoring sulfur atom that chemically contacts to the surface of metal substrates to the distal hydrogen atom. ${ }^{\mathrm{b}}$ Length $(d)$ calculated based on the number of non-hydrogen atoms in the backbone of the molecule. ${ }^{c}$ Values of J0 are extrapolation of the linear plots of Figure 2, and are included only for completeness. Because all chains (other than n-alkanes) are heterogeneous, this extrapolation is not meaningful. ${ }^{\mathrm{d}}$ Ref. $23 .{ }^{\mathrm{e}}$ Ref. 17. ${ }^{\mathrm{f}}$ Number of non-hydrogen atoms for each phenyl ring in the backbone consider $4 .{ }^{\mathrm{g}}$ Ref. 21. 
Table 2. Orbital energies (eV) for the spin-up (alpha) molecular orbitals (MO) localized on A) the sulfur atom, B) the lone pair of oxygen and the $\mathrm{C}-\mathrm{H}$ bond, and $\mathrm{C}$ ) $\mathrm{C}-\mathrm{C}$ bond for thiol-terminated oligoethylene glycol and dodecanethiolate on $\mathrm{Au}_{10}$ clusters. The results for the whole series of compounds $\left(\mathrm{Au} / \mathrm{S}\left(\mathrm{CH}_{2} \mathrm{CH}_{2} \mathrm{O}\right)_{\mathrm{n}} \mathrm{CH}_{3} ; \mathrm{n}=1-7\right)$ are shown in the Supporting Information.

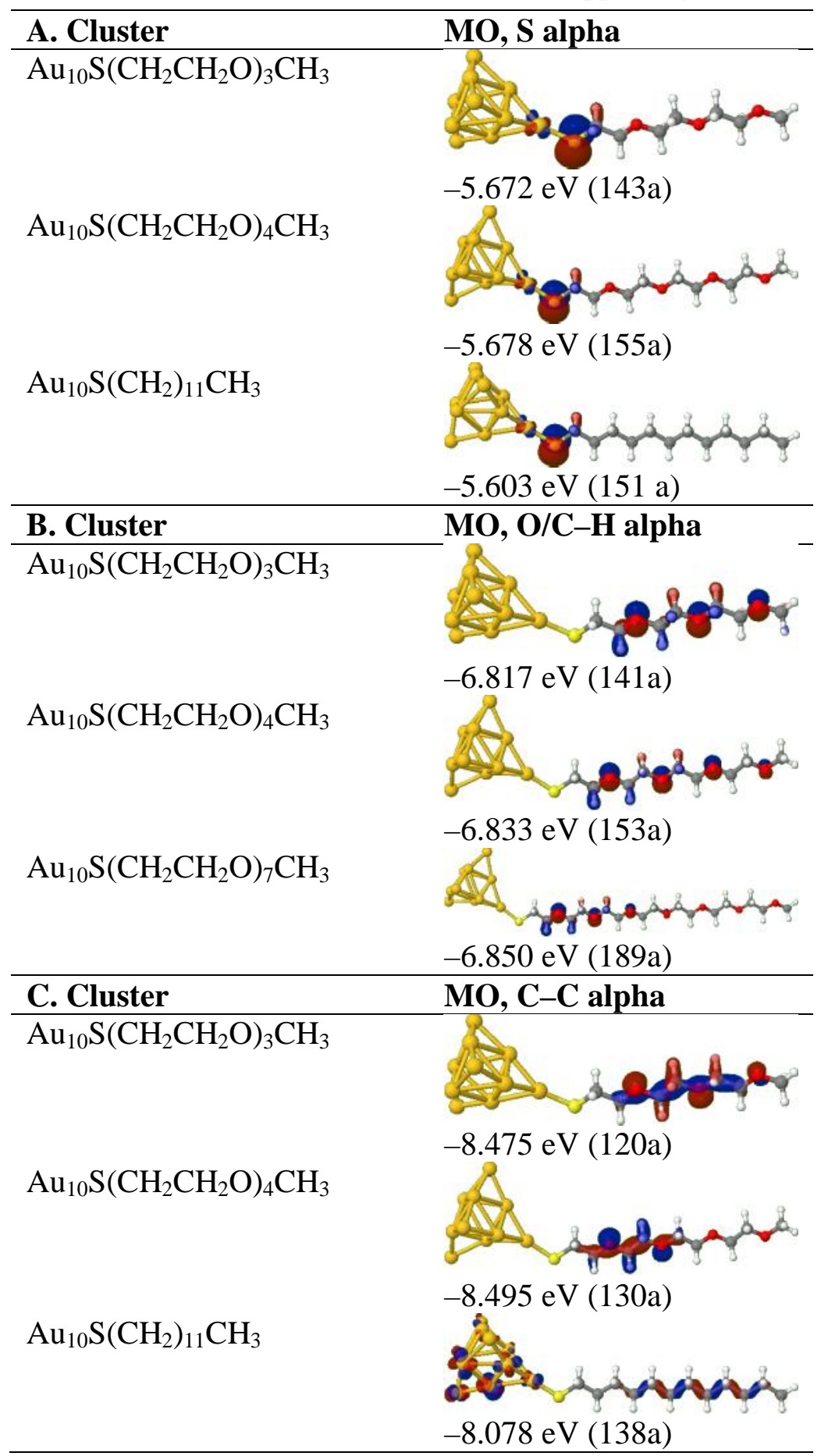


Table 3. Summary of the Superexchange tunneling parameters, $\varepsilon_{0}$ and $V$, for oligoethylene-, oligopropylene-, and oligobutyl glycols

\begin{tabular}{l|l|l}
\hline Cluster & $\boldsymbol{\varepsilon}_{\mathbf{0}}(\mathbf{e V})$ & $\boldsymbol{V}(\mathbf{e V})$ \\
\hline $\mathrm{Au}_{10} \mathrm{~S}\left(\mathrm{CH}_{2} \mathrm{CH}_{2} \mathrm{O}\right)_{n} \mathrm{CH}_{3}$ & $-6.99 \pm 0.007$ & $-0.09 \pm 0.005$ \\
$\mathrm{Au}_{10} \mathrm{~S}\left(\mathrm{CH}_{2} \mathrm{CH}_{2} \mathrm{CH}_{2} \mathrm{O}\right)_{n} \mathrm{CH}_{3}$ & $-6.91 \pm 0.004$ & $-0.09 \pm 0.003$ \\
$\mathrm{Au}_{10} \mathrm{~S}\left(\mathrm{CH}_{2} \mathrm{CH}_{2} \mathrm{CH}_{2} \mathrm{CH}_{2} \mathrm{O}\right)_{n} \mathrm{CH}_{3}$ & $-6.85 \pm 0.010$ & $-0.05 \pm 0.010$ \\
\hline
\end{tabular}

\section{REFERENCES}

1. $\quad$ Nitzan, A.; Ratner, M. A. Science 2003, 300, 1384-1389.

2. Thompson, D.; Nijhuis, C. A. Acc. Chem. Res. 2016, 49, 2061-2069.

3. McCreery, R. L.; Yan, H.; Bergren, A. J. Phys. Chem. Chem. Phys. 2013, 15, 1065-1081.

4. Perrin, M. L.; Burzuri, E.; van der Zant, H. S. J. Chem. Soc. Rev. 2015, 44, 902-919.

5. Metzger, R. M. Chem. Rev. 2015, 115, 5056-5115.

6. Xiang, D.; Wang, X.; Jia, C.; Lee, T.; Guo, X. Chem. Rev. 2016, 116, 4318-4440.

7. Vilan, A.; Aswal, D.; Cahen, D. Chem. Rev. 2017, DOI: 10.1021/acs.chemrev.6b00595.

8. Fracasso, D.; Muglali, M. I.; Rohwerder, M.; Terfort, A.; Chiechi, R. C. J. Phys. Chem. C 2013, 117, 11367-11376.

9. $\quad$ Sangeeth, C. S. S.; Wan, A.; Nijhuis, C. A. J. Am. Chem. Soc. 2014, 136, 11134-11144.

10. Sayed, S. Y.; Fereiro, J. A.; Yan, H. J.; McCreery, R. L.; Bergren, A. J. Proc. Natl. Acad. Sci. USA 2012, 109, 11498-11503.

11. Bergren, A. J.; McCreery, R. L.; Stoyanov, S. R.; Gusarov, S.; Kovalenko, A. J. Phys. Chem. C 2010, 114, 15806-15815.

12. Yan, H.; Bergren, A. J.; McCreery, R. L. J. Am. Chem. Soc. 2011, 133, 19168-19177.

13. Bergfield, J. P.; Ratner, M. A. Phys. Status Solidi B 2013, 250, 2249-2266.

14. McCreery, R. L.; Bergren, A. J. Adv. Mater. 2009, 21, 4303-4322.

15. Yoon, H. J.; Shapiro, N. D.; Park, K. M.; Thuo, M. M.; Soh, S.; Whitesides, G. M. Angew. Chem. Int. Ed. 2012, 51, 4658-4661.

16. Yoon, H. J.; Bowers, C. M.; Baghbanzadeh, M.; Whitesides, G. M. J. Am. Chem. Soc. 2014, 136, 16-19.

17. Bowers, C. M.; Rappoport, D.; Baghbanzadeh, M.; Simeone, F. C.; Liao, K. C.; Semenov, S. N.; Zaba, T.; Cyganik, P.; Aspuru-Guzik, A.; Whitesides, G. M. J. Phys. Chem. C 2016, 120, 1133111337.

18. Salomno, A.; Böcking, T.; Goding, J. J.; Cahen, D. Nano Lett. 2006, 6, 2873-2876.

19. Wold, D. J.; Haag, R.; Rampi, M. A.; Frisbie, C. D. J. Phys. Chem. B 2002, 106, 2813-2816.

20. Ho Choi, S.; Kim, B.; Frisbie, C. D. Science 2008, 320, 1482-1486.

21. Baghbanzadeh, M.; Bowers, C. M.; Rappoport, D.; Zaba, T.; Gonidec, M.; Al-Sayah, M. H.; Cyganik, P.; Aspuru-Guzik, A.; Whitesides, G. M. Angew. Chem. Int. Ed. 2015, 54, 14743-14747.

22. Simeone, F. C.; Yoon, H. J.; Thuo, M. M.; Barber, J. R.; Smith, B.; Whitesides, G. M. J. Am. Chem. Soc. 2013, 18131-18144.

23. Bowers, C. M.; Liao, K. C.; Zaba, T.; Rappoport, D.; Baghbanzadeh, M.; Breiten, B.; Krzykawska, A.; Cyganik, P.; Whitesides, G. M. ACS Nano 2015, 9, 1471-1477.

24. Sepunaru, L.; Refaely-Abramson, S.; Lovrincic, R.; Gavrilov, Y.; Agrawal, P.; Levy, Y.; Kronik, L.; Pecht, I.; Sheves, M.; Cahen, D. J. Am. Chem. Soc. 2015, 137, 9617-9626.

25. Sangeeth, C. S.; Wan, A.; Nijhuis, C. A. Nanoscale 2015, 7, 12061-12067. 
26. Vilan, A.; Cahen, D. Chem. Rev. 2017, DOI: 10.1021/acs.chemrev.6b00746.

27. Wierzbinski, E.; Yin, X.; Werling, K.; Waldeck, D. H. J. Phys. Chem. B 2013, 117, 4431-4441.

28. Xie, Z.; Bâldea, I.; Oram, S.; Smith, C. E.; Frisbie, C. D. ACS Nano 2017, 11, 569-578.

29. Song, H.; Kim, Y.; Jang, Y. H.; Jeong, H.; Reed, M. A.; Lee, T. Nature 2009, 462, 1039-1043.

30. Bruot, C.; Hihath, J.; Tao, N. J. Nat. Nanotechnol. 2012, 7, 35-40.

31. Guo, C. L.; Yu, X.; Refaely-Abramson, S.; Sepunaru, L.; Bendikov, T.; Pecht, I.; Kronik, L.;

Vilan, A.; Sheves, M.; Cahen, D. Proc. Natl. Acad. Sci. USA 2016, 113, 10785-10790.

32. Kumar, S.; van Herpt, J. T.; Gengler, R. Y. N.; Feringa, B.; Rudolf, P.; Chiechi, R. C. J. Am. Chem. Soc. 2016, 138, 12519-12526.

33. Song, P.; Yuan, L.; Roemer, M.; Jiang, L.; Nijhuis, C. A. J. Am. Chem. Soc. 2016, 138, 57695772.

34. Simmons, J. G. J. Appl. Phys. 1963, 34, 1793-1803.

35. Choi, S. H.; Frisbie, C. D. J. Am. Chem. Soc. 2010, 132, 16191-16201.

36. Jiang, L.; Sangeeth, C. S. S.; Wan, A.; Vilan, A.; Nijhuis, C. A. J. Phys. Chem. C 2015, 119, 960-969.

37. Kosloff, R.; Ratner, M. A. Isr. J. Chem. 1990, 30, 45-58.

38. Nitzan, A. Annu. Rev. Phys. Chem. 2001, 52, 681-750.

39. McConnell, H. J. Chem. Phys. 1961, 35, 508.

40. Joachim, C.; Ratner, M. A. Proc. Natl. Acad. Sci. USA 2005, 102, 8801-8808.

41. Gray, H. B.; Winkler, J. R. Proc. Natl. Acad. Sci. USA 2005, 102, 3534-9.

42. Weigend, F. Phys. Chem. Chem. Phys. 2006, 8, 1057-1065.

43. Weiss, E. A.; Chiechi, R. C.; Kaufman, G. K.; Kriebel, J. K.; Li, Z. F.; Duati, M.; Rampi, M. A.; Whitesides, G. M. J. Am. Chem. Soc. 2007, 129, 4336-4349.

44. Vanderah, D. J.; Valincius, G.; Meuse, C. W. Langmuir 2002, 18, 4674-4680.

45. Vanderah, D. J.; Arsenault, J.; La, H.; Gates, R. S.; Silin, V.; Meuse, C. W.; Valincius, G. Langmuir 2003, 19, 3752-3756.

46. Palik, E. D., Handbook of Optical Constants of Solids. Academic Press: Orlando, 1985; p xviii, $804 \mathrm{p}$.

47. Weiss, E. A.; Kaufman, G. K.; Kriebel, J. K.; Li, Z.; Schalek, R.; Whitesides, G. M. Langmuir 2007, 23, 9686-9694.

48. $\quad$ Bowers, C. M., Liao, K. C., Yoon, H. J., Rappoport, D., Baghbanzadeh, M., Simeone, F. C, Whitesides, G. M. Nano Lett. 2014, 14, 3521-3526.

49. Engelkes, V. B.; Beebe, J. M.; Frisbie, C. D. J. Am. Chem. Soc. 2004, 126, 14287-14296.

50. Baghbanzadeh, M.; Simeone, F. C.; Bowers, C. M.; Liao, K. C.; Thuo, M.; Miller, M. S.;

Carmichael, T. B.; Whitesides, G. M. J. Am. Chem. Soc. 2014, 136, 16919-16925.

51. Liao, K.-C., Yoon, H.J., Bowers, C.M., Simeone, F.C., Whitesides, G.M Angew. Chem. Int. Ed. 2014, 53, 3889-3893.

52. Love, J. C.; Estroff, L. A.; Kriebel, J. K.; Nuzzo, R. G.; Whitesides, G. M. Chem. Rev. 2005, 105, 1103-1169.

53. Cheng, T. L.; Chuang, K. H.; Chen, B. M.; Roffler, S. R. Bioconjug. Chem. 2012, 23, 881-899.

54. Costner, E. A.; Long, B. K.; Navar, C.; Jockusch, S.; Lei, X.; Zimmerman, P.; Campion, A.;

Turro, N. J.; Willson, C. G. J. Phys. Chem. A 2009, 113, 9337-9347.

55. Becke, A. D. J. Chem. Phys. 1993, 98, 5648-5652.

56. Weigend, F.; Ahlrichs, R. Phys. Chem. Chem. Phys. 2005, 7, 3297-3305. 